\title{
SOURCES AND BIBLIOGRAPHY
}

\author{
MANUSCRIPTS
}

\section{Bibliothèque nationale de France}

Correspondence from reign of Charles IX

MS Fr. 15553, year 1571, especially fols $197 \mathrm{ff}$, letters of Geoffroy de Caumont on his flight from the faubourg Saint-Germain, 24 Aug. 1572.

MS Fr. 15554, Jan.-June 1572, especially fols $124 \mathrm{r}-127 \mathrm{r}$, letter from president Lagebaston to Charles IX, 7 Oct. 1572.

MS Fr. 15555, July-Dec. 1572.

MS Fr. 15556, Jan.-Feb. 1573.

MS Fr. 15557, March-May 1573.

MS Fr. 15558, June-Dec. 1573.

MS Fr. 15559, 1574.

Correspondence and other original documents on wars of religion

MS Fr. 3193, especially fols 68-69: 'memorandum on the words spoken by Admiral Coligny in the King's study in August 1572.'

MS Fr. 3209, especially fols 66 and 79: letters from duc de Longueville, governor of Picardy, to M. d'Humières, 28 Aug. 1572 and 16 Oct. 1572.

MS Fr. 3256, especially fol. 59, royal circular, 2 Sept. 1572.

MS Fr. 3951, especially fol. 5, letter of Charles IX to Schomberg, 13 Sept. 1572, and fol. 142, Charles IX to Cardinal Lorraine, 31 July 1572.

MS Fr. 16104, especially fol. 45, circular letter from Charles IX to provincial governors, 4 May 1572.

MS 500 Colbert, MS. 7, especially fol. 425, mémoire for sieur de Changy on mission to the sieur de Villiers, 27 Aug. 1572; fols 449 and 461, records of campaigns to convert Protestant nobles of Metz and Champagne.

MS 500 Colbert, MS. 24, especially fol. 415, Coligny's letter to Charles IX, La Rochelle, 8 May 1571.

Narrative accounts

MS Fr. 17309, fols 56ff, Dessein de ceulx qui soubz le nom et autorité de Sa Majesté ont faict le massacre (probably written in Autumn 1573 or early 1574 by a Protestant or a Catholic close to the duc d'Alençon). 
MS Fr. 17529, fols 1ff, Discours particulier où est amplement descrit et blasmé le massacre de la St-Barthélemy, and fols $176 \mathrm{ff}$, Discours du Roy Henry troisiesme a un personnage d'honneur [...] des causes et motifs de la Sainct Barthelemy (an apocryphal account, probably emanating from the Gondi family entourage, and published in 1623 with a view to exonerating the comte de Retz).

Embassies and diplomatic papers

MS Fr. 16040, Roman embassy of M. de Ferrals, 1572-73.

MS Fr. 18895, fols 205ff, Pomponne de Bellièvre, Proposition faite aux Suisses [...] sur la mort de Monsieur l'admiral de Colligny et journee de St Barthelemy.

MS Fr. 16104, Spanish embassy of M. de Saint-Gouard, Jan.-Dec. 1572.

MS Fr. 5172, Jean de Morvillier's Memoires d'Estat, especially fol. 2r, 'Charles IX's proposals to princes and lords assembled in his presence in 1571'; and fol. 6ff, 'instruction sent to provincial governors', 3 Nov. 1572.

Other documents

MS Fr. 16250, Pierre de Blanchefort, Compte de mes actions et recouil depuis la proclamation des Estats tenus à Blois nottez par mois et jours.

MS Fr. 18288, Vie de messire Jehan de Morvillier, by Nicolas Le Fèvre, sieur de Lezeau.

MS. Nouvelles acquisitions françaises, 7178, fols 36r-47r, articles and petitions from the 'so-called reformed religion' of Languedoc assembled at Montauban.

\section{Archivio General de Simancas}

(Microfilm in Archives Nationales, Paris)

K1524-K1536: correspondence between ambassador Diego de Zuñiga, Duke of Alba and Philip II, 1572-74, especially K1524, no. 78, relation of the death of the Admiral and other heretics in France, and no. 79, what Juan de Olaegui, secretary of the ambassador don Diego de Zuñiga ... reported on matters in the French court; and K1530, nos 19, 20, 21 and 29: Zuñiga's letters of 20, 23 (erroneously dated 22), 26 (erroneously dated 23) and 31 Aug. 1572.

\section{Bibliothèque publique universitaire de Genève}

MS Fr. 90: correspondence of Pierre Forget de Fresnes, France's envoy to the Duke of Savoy.

\section{PRINTED SOURCES}

Arbaleste, Charlotte, Mémoires de Madame de Mornay, ed. Madame de Witt, 2 vols, Paris, 1868.

Archives ou Correspondance inédite de la maison d'Orange-Nassau, ed. Guillaume Groen Van Prinsterer, 1st series, 8 vols, Leiden, 1835-96.

Archivo Documental Español. Negociaciones con Francia, 1557-1568, 11 vols, Madrid, 1950-6o.

Aubigné, Agrippa d', Histoire Universelle, ed. André Thierry, Geneva, 11 vols, 19812000. 
—, Les Tragiques, ed. Jean-Raymond Fanlo, 2 vols, Paris, 2003.

Baif, Jean-Antoine de, Euvres en rime, ed. Charles Marty-Laveaux, 5 vols, Paris, 1881-90, reprint Geneva, 1966.

Barbiche, Bernard, ed., L'Édit de Nantes et ses antécedents, electronic edition of the edicts of pacification of the wars of religion: http://elec.enc.sorbonne.fr.

Barclay, William, De regno et regali potestate adversus Buchanan, Brutum, Boucherium et reliquos monarchomaquos libri sex, Paris, 1600.

Bellaud, Jean-Baptiste, Oraison funebre du trespas du Roy treschrestien Charles Neufiesme, Paris, 1574.

Belleforest, François de, Discours sur l'heur des presages advenuz de nostre temps, signifiantz la felicité de nostre Roy Charles neufiesme tres chrestien, Paris, 1572.

-, Discours sur les rebellions auquel est contenu quelle est la misere qui accompagne les trahistres seditieux et rebelles et les recompenses qui les suivent selon leurs rebellions, avec un arraisonnement fort proffitable sur l'infelicité qui suit ordinairemenet les grans, Paris, 1572.

Benoist, René, Advertissement du moyen par lequel aisément tous troubles et differens tant touchant la Croix, de laquelle y a si grande et si dangereuse altercation en ceste ville de Paris, que autres concernans la Religion, Paris, 1571 (reprinted in Simon Goulart, ed., Memoires de l'Estat de France sous Charles IX, 2nd ed., Middleburg, 1578, vol. 1, fols 88r-95r).

Bèze, Théodore de, Correspondance, ed. Alain Dufour and Béatrice Nicollier, vol. 13 (1572), Geneva, 1988; vol. 14 (1573), and vol. 15 (1575).

—, Du Droit des magistrats sur leur subjets, ed. Robert M. Kingdon, Geneva, 1971.

[Bèze, Théodore de], Histoire ecclésiastique des Églises réformées de France, Geneva, 1580, ed. Jean-Guillaume Baum and Edouard Cunitz, 3 vols, Paris, 1883-89.

Bodin, Jean, Les Six Livres de la République, Paris, Fayard, 1986 (reprint of 1593 edition).

Botzheim, Johann Wilhelm von, 'La Saint-Barthélemy à Orléans racontée par Johann Wilhelm de Botzeim, étudiant allemand, témoin oculaire' [trans. from Latin by Charles Read], in BSHPF, 21 (1872), pp. 345-392.

Bouquet, Simon, Brief et sommaire recueil de ce qui a esté faict et de l'ordre tenuë a la joyeuse et triumphante Entrée de Charles IX de ce nom en sa bonne ville et cité de Paris, Paris, 1572.

Bourdeille, Pierre de, seigneur de Brantôme, Euvres complètes, ed. Ludovic Lalanne, 11 vols, Paris, 1864-82.

Boutaric, Edgar, 'La Saint-Barthélemy d'après les archives du Vatican', Bibliothèque de l'École des Chartes, 23 (1862), pp. 1-27.

Briefve instruction de tout ce qui a passé en la ville de Thoulouze depuis l'emprisonnement faict de ceulx de la nouvelle pretendue religion (Nov. 1572), in Dom Devic and Dom Vaissète, Histoire générale de Languedoc (Paris-Toulouse, 2004-6), vol. 12, col. 1028, preuve 316.

Budé, Guillaume, L'Institution du Prince, ed. Claude Bontemps, in Claude Bontemps et alii, Le Prince dans la France des xvie et xvii siècles (Paris, 1966), pp. 77-139.

Burin, Pierre, Response à une epistre commencant Seigneur Elvide, où est tracté des massacres faits en France en l'an 1572, Bâle, 1574.

Cabié, Edmond, Guerres de Religion dans le Sud-Ouest de la France et principale- 
ment dans le Quercy d'après les papiers des seigneurs de Saint-Sulpice, de 1561 à 159o, 2 vols, Paris-Toulouse, 1906.

Calendar of State Papers, Foreign Series, Reign of Elizabeth, 1566-1577, vol. 3 (15721574), London, 1876.

Calendar of State Papers relating to English Affairs, preserved essentially at Rome, in the Vatican Archives and Library, vol. 2, Elizabeth, 1572-1578, ed. James M. Rigg, London, 1926.

Calvin, Jean, Institution de la religion chrestienne, ed. Jean-Daniel Benoît, 4 vols, Paris, 1961.

Capilupi, Camillo, Lo Stratagema di Carlo IX, re di Francia, contro gli Ugonotti, rebelli di Dio et suoi, Roma, 1572; anonymous French trans., Le Stratagème de Charles IX, Roy de France, contre les Huguenots rebelles à Dieu et luy, reprinted in Louis Cimber and Charles Danjou, eds, Archives curieuses de l'Histoire de France, 1st series, vol. 7 (Paris, 1835), pp. 401-471.

Caumont, Jacques-Nompar de, Mémoires authentiques de Jacques-Nompar de Caumont, duc de La Force, ed. Edouard Lelièvre, Marquis de La Grange, 4 vols, Paris, 1843.

Chantelouve, François, La tragedie de feu Gaspar de Colligni, jadis amiral de France: contenant ce qui advint à Paris le 24 aoust 1572, [n.p., n.d., 1575], ed. Keith Cameron, Exeter, 1971.

Charpentier, Pierre, Lettre de Pierre Charpentier jurisconsulte addressée à François Portes Candiois, par laquelle il monstre que les persécutions des Églises de France sont advenues non par la faulte de ceux qui faisoient profession de la religion, mais de ceux qui nourrissoient les factions et conspirations qu'on appelle la Cause, n.p., n.d. [n.p., n.d., 1572].

Coras, Jean de, Question politique: s'il est licite aux subjects de capituler avec leur prince, ed. Robert M. Kingdon, Geneva, 1989.

Correspondance de Philippe II sur les affaires des Pays-Bas, publiée d'après les originaux conservés dans les archives royales de Simancas, ed. Louis Gachard, 5 vols, Brussels, 1848-79.

Correspondance diplomatique de Bertrand de Salignac de La Mothe-Fénelon, ed. Charles Purton Cooper, 7 vols, Paris-London, 1838-40 (vol. 7 is a supplement, subtitled Lettres adressées de la Cour à l'ambassadeur).

Correspondance du nonce en France Antonio Maria Salviati (1572-1578), ed. Pierre Hurtubise and Robert Toupin, 2 vols, Rome, École française de Rome, 1975.

Correspondance du roi Charles IX et du sieur de Mandelot, gouverneur de Lyon, pendant l'année 1572, ed. Paulin Paris, Paris, 1830.

Déclaration de Henri de Bourbon aujourd'huy troisiesme Prince du sang de France, prince de Condé, accompaigné de plusieurs gentilshommes de l'une et l'autre Religion, La Rochelle, 1574.

Déclaration de Monseigneur François, fils et frère de Roy, duc d'Alençon, n. p., 1575.

Déclaration et protestation de Monseigneur de Dampville, maréchal de France, [13 Nov. 1574] in Dom Devic and Dom Vaissète, Histoire générale de Languedoc, Paris-Toulouse (2004-6), vol. 12, cols 1105-1111, preuve 336.

Désiré, Artus, La Singerie des Huguenots marmots et guenons de la nouvelle derrision theodobeszienne, Paris, 1574. 
Desportes, Joachim, Discours sommaire du règne de Charles neufiesme Roy de France Tres-Chrestien, ensemble de sa mort, et d'aucuns de ses derniers propos, Paris, s.d. [1574].

'Deux lettres de couvent à couvent écrites de Paris pendant le massacre de la SaintBarthélemy (25 et 26 août) par Joachim Opser de Wyl, jésuite sous-proviseur du collège de Clermont à Paris', BHSPF, 8 (1859), pp. 284-294.

Digges, Dudley, ed., The Compleat Ambassador, or Two Treaties of the Intended Marriage of Queen Elizabeth of glorious memory comprised in Letters of Negociation of sir Francis Walsingham, London, 1655.

Discours du Roy Henry troisiesme a un personnage d'honneur et de qualité, estant près de Sa Majesté, des causes et motifs de la Sainct Barthelemy, in Mémoires d'Estat de Nicolas de Neufville, seigneur de Villeroy (Paris, 1623), pp. 68-69, reprinted in Claude Petitot, ed., Collection de mémoires relatifs à l'histoire de France, 1st series, vol. 44 (Paris, 1824), pp. 496-510.

Discours merveilleux de la vie, actions et déportements de Catherine de Médicis [1575 and 1576], ed. Nicole Cazauran, Geneva, 1995.

Discours politiques des diverses puissances establies de Dieu au monde, du gouvernement legitime d'icelles, et de ceux qui y sont assujettis, published in Simon Goulart, Mémoires de l'Estat de France sous Charles neuviesme, $2{ }^{\mathrm{e}}$ ed. (Middleburg, 1578), vol. 3, fols 203v-296r.

Discours sur la mort de Gaspart de Coligny qui fut admiral de France, by I. S. P., Paris, 1572.

Dorat, Jean, Euvres poétiques, ed. Charles Marty-Laveaux, Paris, 1875, reprint Geneva, 1974.

[Du Faur de Pibrac, Guy], Traduction d'une Epistre latine d'un excellent personnage de ce Royaume, Paris, 1573.

Du Faur de Pibrac, Guy, Les Quatrains. Les Plaisirs de la vie rustique et autres poésies, ed. Loris Petris, Geneva, 2004.

[Du Haillan, Bernard de Girard, seigneur], Discours sur les causes de l'execution faicte es personnes de ceux qui avoyent conjuré contre le Roy et son Estat, Paris, 1572.

Du Part, Antoine, Déploration de la France sur le trespas du Tres Chrestien Roy Charles IX, Paris, 1574.

Fabre, Pierre, Response au cruel et pernicieux conseil de Pierre Charpentier, chiquaneur, tendant à fin d'empescher la paix et nous laisser la guerre, traitté dans lequel on apprendra en quel cas il est permis à l'homme chrestien de porter les armes. Traduit du latin, n.p., n.d. [1575].

Gachard, Louis, La Bibliothèque nationale à Paris. Notices et extraits des manuscrits qui concernent l'histoire de Belgique, 2 vols, Brussels, 1875-77.

Gaches, Jacques, Mémoires sur les guerres de religion à Castres et dans le Languedoc, 1555-1610, ed. Charles Pradel, Paris, 1879 (reprint Geneva, 1970).

Garrisson, Janine, ed., L’édit de Nantes, Biarritz, 1997.

Gassot, Jules, Sommaire Mémorial (1555-1623), ed. Pierre Champion, Paris, 1934.

Geizkofler, Luc, Mémoires de Luc Geizkofler, tyrolien (1550-1620), ed. Edouard Fick, Geneva, 1892.

[Gentillet, Innocent], Briève Remonstrance à la noblesse de France sur le faict de la Déclaration de Monseigneur le duc d'Alençon, n. p., 1576. 
Gentillet, Innocent, Discours sur les moyens de bien gouverner et maintenir en bonne paix un Royaume ou autre Principauté [...] contre Nicolas Machiavel Florentin, 1576, ed. C. Edward Rathé, Geneva, 1968.

Goulart, Simon, ed., Mémoires de l'Estat de France sous Charles neuviesme, $2^{\mathrm{e}}$ ed., 3 vols, Middleburg, 1578.

Haton, Claude, Mémoires, ed. Laurent Bourquin, 4 vols, Paris, 2001-7.

[Hotman, François], De furoribus gallicis, horrenda et indigna amiralii Castillionei, nobilium atque illustrium virorum caede, scelerata ac inaudita piorum strage passim edita per complures civitates [...] Ernesto Varamundo Frisio auctore, Edinbugh 1573; anonymous French trans., Histoire des massacres et horribles cruautez commises en la personne de Messire Gaspar de Colligny et autres seigneurs gentils-hommes, le 24 jour d'aoust 1572 et autres suivans. Traduite en françois et augmentée de quelque particularitez omises en l'exemplaire latin, n.p., 1573.

[Hotman, François], Gasparis Colinii Castellonii, magni quondam Franciae amiralii, vita, n.p., 1575; anonymous French trans., La Vie de messire Gaspar de Colligny, seigneur de Chastillon, admiral de France, Leiden, 1643, ed. Émile Telle, Geneva, 1987.

Hotman, François, Francogallia, n.p., ed. Ralph Giesey and John Salmon, Cambridge, 1972; French trans. attributed to Simon Goulart, La Gaule françoise de François Hotman jurisconsulte, Cologne, 1574, ed. Antoine Leca, Aix-Marseille, 1991.

Jeannin, Pierre, Les Négociations de Monsieur le président Jeannin, Paris, 1656.

Kervyn de Lettenhove, Joseph, ed., Documents inédits relatifs à l'histoire du xvi siècle, Brussels, 1883 .

L'Estoile, Pierre de, Mémoires pour servir à l'histoire de France depuis 1515 jusqu'en 1574, ed. André Martin, as appendix to l'Estoile, Journal pour le règne de Henri IV et le début du règne de Louis XIII, Paris, Gallimard, 1960, pp. 447-483.

-, Registre-Journal du règne de Henri III, ed. Madeleine Lazard and Gilbert Schrenck, 6 vols, Geneva, 1992-2003.

L'Histoire de la mort que le R. P. Edmond Campion, Prestre de la compagnie du nom de Jesus, et d'autres ont souffert en Angleterre pour la foy Catholique et Romaine, Paris, 1582.

L'Hospital, Michel, Discours pour la majorité de Charles IX et trois autres discours, ed. Robert Descimon, Paris, 1993.

-, Euvres complètes, ed. Pierre Joseph Spiridion Duféy, 5 vols, Paris, 1824-36, reprint Geneva, 1968.

La Ferrière, Hector de, Le XVI siècle et les Valois, d'après les documents inédits $d u$ British Museum et du Record Office, Paris, 1879, p. 419.

La Fosse, Jehan de, Les 'Mémoires' d'un curé de Paris (1557-159o), ed. Marc Venard, Geneva, 2004.

La France-Turquie, c'est-à-dire conseils et moyens tenus par les ennemis de la Couronne de France pour reduire le royaume en tel estat que la tyrannie turquesque, Orléans, 1576. (This collection contains the L'Antipharmaque du chevalier Poncet [Paris, 1575], and the Lunettes de christal de roche par lesquels on veoyt clairement le chemin tenu pour subjuguer la France a mesme obeissance que la Turquie, n.p., n.d.) 
La Huguerye, Michel, Mémoires inédits 1570-1602, ed. Alphonse de Ruble, 3 vols, Paris, $1877-80$.

La Noue, François de, Discours politiques et militaires, ed. F. E. Sutcliffe, Geneva, 1967.

La Saint-Barthélemy devant le Sénat de Venise. Relations des ambassadeurs Giovanni Michiel et Sigismondo Cavalli, published by William Martin, Paris, 1872.

Le Politique: Dialogue traitant de la puissance, authorité et du devoir des Princes: des divers gouvernement: jusques où l'on doit supporter la tyrannie: si en une oppression extreme il est loisible aux sujets de prendre les armes pour defendre leur vie et liberté: quand, par qui, et par quel moyen cela se peut et doit faire, published by Simon Goulart, Mémoires de l'Estat de France sous Charles neuviesme, $2^{\mathrm{e}}$ ed. (Middleburg, 1578), vol. 3 , fols $61 \mathrm{r}-116 \mathrm{v}$.

Le Réveille-Matin des François et de leurs voisins, composé par Eusèbe Philadephe Cosmopolite en forme de dialogues, 2 vols, Edinburgh, 1574; facsimile reprint, Paris, 1977.

Le Roy, Louis, Les Politiques d'Aristote. Traduictes de grec en françois, avec expositions prises des meilleurs auteurs, specialement d'Aristote mesme, et de Platon conferez ensemble, Paris, 1568.

Le Trespas et obseques du Treschrestien Roy de France, Charles neufiesme de ce nom, Lyon, 1574.

Le Vray Discours des derniers propos memorables et trespas du feu Roy de tres bonne memoire Charles neufiesme, Paris, 1574.

Les Regretz et lamentations de tresillustre et vertueuse princesse Elisabeth d'Autriche sur la mort et trespas du Roy Charles neufiesme son espoux, n'agueres decedé. Par A. de la T. D., Paris, 1574.

Lettres de Catherine de Medicis, ed. Hector de La Ferrière and Gustave Baguenault de Puchesse, 10 vols, Paris, 1880-1909.

Lettres de Charles IX à M. de Fourquevaux, ambassadeur en Espagne, 1565-1572, ed. Charles Douais, Montpellier, 1897.

Lettres du cardinal Charles de Lorraine (1525--1574), ed. Daniel Cuisiat, Geneva, 1998.

Lettres inédites de Jacques Faye et de Charles Faye, ed. Eugène Halphen, Paris, 1880.

Marguerite de Valois, Correspondance, ed. Éliane Viennot, Paris, 1998.

—, Mémoires et autres écrits, ed. Éliane Viennot, Paris, 1999.

Memoires de la troisieme guerre civile, 1576, published in Simon Goulart, Mémoires de l'Estat de France sous Charles neuviesme, $2^{\mathrm{e}}$ ed., Middleburg, 1578, vol. 3.

Mémoires des occasions de la guerre, appellée Le Bien-Public, rapportez à l'estat de la guerre présente, n. p., 1567.

Mémoires et instructions pour les ambassadeurs, ou lettres et négociations de Walsingham, ministre et secrétaire d'État sous Élisabeth, reine d'Angleterre, traduit de l'anglois, Amsterdam, 1700.

Mergey, Jean de, Mémoires, ed. Michaud and Poujoulat, Mémoires pour servir à l'histoire de France, vol. 9 (Paris, 1838), pp. 557-58o.

Mieck, Ilja, ed., Toleranzdikt und Bartholomäusnacht. Französiche Politik und europäische Diplomatie, 1570-1572, Göttingen, 1969. 
Monluc, Blaise de, Commentaires, 1521-1576, ed. Paul Courteault, Paris, 1962.

[Monluc, Jean de], Vera et brevis descriptio tumultus postremi Gallici Lutetiani, Cracow, 1573.

Monluc, Jean de, Harangue faicte et prononcée de la part du Roy Très-Chrestien le dixième jour du mois d'avril 1573, Paris, 1573.

-, Défense pour maintenir le tresillustre Duc d'Anjou contre les calomnies de quelques malveillans, in Simon Goulart, Mémoires de l'Estat de France sous Charles neuviesme, $2^{\mathrm{e}}$ ed. (Middleburg, 1578), vol. 2, fols 61v-69v.

Montaigne, Michel de, Les Essais, ed. Pierre Villey, 3 vols, Paris, 1922-23; reprint Paris, 1988.

Négociations diplomatiques de la France avec la Toscane, ed. Abel Desjardins, 6 vols, Paris, 1859-86.

Pape, Jacques, Mémoires de Jacques Pape, seigneur de Saint-Auban, ed. Michaud and Poujoulat, Paris, vol. 11 (Paris, 1838), pp. 497-514.

Pasquier, Étienne, Les Lettres, in Euvres (Amsterdam, 1723), vol. 2, reprint Geneva, 1971.

-, Lettres historiques pour les années 1556-1594, ed. Dorothy Thickett, Geneva, 1966.

-, Les Recherches de la France, ed. Marie-Madeleine Fragonard and François Roudaut, 3 vols, Paris, 1996.

Pithou de Chamgobert, Nicolas, Chronique de Troyes et de la Champagne (15241594), ed. Pierre-Eugène Leroy, 3 vols, Reims, 1998-20oo.

Portes, François, Response de François Portus Candiot aux lettres diffamatoires de Pierre Charpentier advocat. Pour l'innocence des fidèles serviteurs de Dieu et obeissans subjets du Roy, massacrez le 24. jour d'Aoust 1572, appellez factieux par ce plaidereau. Traduicte nouvellement de latin en françois, n.p., 1574.

Recueil des choses memorables avenues en France sous le regne de Henri II, François II, Charles IX, Henri III et Henri IV [sometimes attributed to Jean de Serres, but more probably to Simon Goulart], 1595; ${ }^{\mathrm{e}}$ ed., n.p., 1598.

Recueil général des anciennes lois françaises, ed. F. A. Isambert, A. J. Jourdan and Decrusy, 29 vols (Paris, 1821-33), vol. 14 (July 1559-May 1574).

Registres des délibérations du Bureau de la Ville de Paris, 15 vols (Paris, 1883-1921); vol. 6, 1568-1572, ed. Paul Guérin [1891], and vol. 7, 1572-1576, ed. François Bonnardot, [1893].

[Régnier de la Planche, Louis], Histoire de l'Estat de France, tant de la république que de la religion, sous le règne de François II (1576), ed. Jean-Alexandre Buchon (Paris, 1836), pp. 203-421.

'Relation de la journée de la Saint-Barthélemy; manuscrit trouvé dans les archives épiscopales de Wiener-Neustadt (Autriche)', Bulletin des sciences historiques, antiquité, philologie, 6 (1826), pp. 226-231.

Relation du massacre de la Saint-Barthélemy (from Simon Goulart, Mémoires de l'Estat de France sous Charles neufiesme vol. 1), in Louis Cimber and Charles Danjou, eds, Archives curieuses de l'histoire de France, 1st series, vol. 7 (Paris, 1835), pp. 1-76.

Résolution claire et facile sur la question tant de fois faicte de la prise des armes par les inférieurs, Bâle, 1575; expanded edition, Reims, 1577. 
Response de Stanislaus Elvidius [Joachim Camerarius?] a l'epistre d'un certain excellent personnage touchant les affaires de France, 1573, published by Simon Goulart, Mémoires de l'Estat de France sous Charles neuviesme, $2^{\mathrm{e}}$ ed. (Middleburg, 1578), vol. 1, fols 636r-655r.

Response de Wolfgang Prisbachius polonois à une harangue soustenant les massacres et brigandages commis en France, French trans. of Latin original published in La Rochelle, 1573, published by Simon Goulart, Mémoires de l'Estat de France sous Charles neuviesme, $2^{\mathrm{e}}$ ed. (Middleburg, 1578), vol. 2, fols $28 \mathrm{v}-47 \mathrm{v}$.

Response de Zacharie Furnesterus, soustenant linnocence et justice de tant de milliers de personnes massacrées au royaume de France, French trans. of original Latin published in 1573, published by Simon Goulart, Mémoires de l'Estat de France sous Charles neuviesme, $2^{\mathrm{e}}$ ed. (Middleburg,1578), vol. 2, fols 7or-95r.

Reuss, Rodolphe, ed., 'Un nouveau récit de la Saint-Barthélemy par un bourgeois de Strasbourg', BSHPF, 22 (1873), pp. 374-381.

Rigal, Jean-Louis, ed., Mémoires d'un calviniste de Millau (Archives historiques du Rouergue, vol. 2), Rodez, 1911.

Ronsard, Pierre de, Le Tombeau du feu Roy Tres-Chrestien Charles IX, prince tres-debonnaire, tres-vertueux et tres-eloquent, Paris, n. d. (1574).

-, Discours des misères de ce temps et Continuation du Discours des misères de ce temps, in Euvres complètes, ed. Jean Céard, Daniel Ménager and Michel Simonin (Paris, 1994), pp. 991-1006.

Sassetti, Tomasso, Brieve Raccontamiento del gran macello fatto nella città di Parigi il vigesimo quarto giorno d'agosto, published by John Tedeschi, in Alfred Soman, ed., The Massacre of St. Bartholomew. Reappraisals and documents (La Haye, 1974), pp. 112-152.

Satyre Ménippée, ou vertu du Catholicon d'Espagne, ed. Charles Read, Paris, Flammarion, 1892.

Saulx-Tavannes, Jean de, Mémoires de Gaspard de Saulx-Tavannes, ed. Michaud and Poujoulat, vol. 8 (Paris, 1838), pp. 19-434.

Serres, Jean de, Commentariorum de statu religionis et reipublicae in regno Galliae, 1571-72 (n. p); expanded edition 1575, 3 parts in one vol.

Sorbin, Arnaud, Histoire contenant un abrégé de la vie, mours, et vertus du Roy Tres-chrestien et debonnaire Charles IX, vrayement piteux, propugnateur de la Foy Catholique et amateur des bons esprits, Paris, 1574.

-, Le Vray resveille-Matin des calvinistes et publicains François: où est amplement discouru de l'auctorité des Princes, et du devoir des sujets envers eux, Paris, 1576.

-, Oraison funebre du tres hault, puissant et tres chrestien Roy de France, Charles IX, piteux et debonnaire, propugnateur de la Foy Catholique et amateur des bons esprits, prononcée en l'Eglise Nostre-Dame en Paris, le XII. de juillet M.D.LXXIIII, Paris, 1574.

Stegmann, André, ed., Edits des guerres de Religion, Paris, 1979.

Sully, Maximilien de Béthune, duc de, Economies royales, eds David Buisseret and Bernard Barbiche, 2 vols, Paris, 1970-88.

Sureau, Hugues, dit des Rosiers, Confession et recognoissance de Hugues Sureau dict du Rosier touchant sa cheute en la Papauté et les horribles scandales par luy commis, Heidelberg, 1573. 
Syrueilh, François de, Journal, 1568-1585, Archives historiques du département de la Gironde, 13 (1871-72), pp. 244-357.

Taix, Guillaume de, Journal de Guillaume de Taix, published by Lalourcé and Duval, Recueil des pièces originales et authentiques concernant la tenue des États généraux (Paris, 1789), vol. 2, pp. 269-271.

Thou, Jacques-Auguste, Histoire universelle depuis 1543 jusqu'en 1607, traduite sur l'edition latine de Londres, 16 vols, London, 1734.

-, Mémoires de Jacques-Auguste de Thou, depuis 1553 jusqu'en 1601, ed. Claude Petitot, vol. 38, Paris, 1823.

Tocsain contre les massacreurs (1577), in Louis Cimber and Charles Danjou, eds, Archives curieuses de l'histoire de France, 1st series, vol. 7 (Paris, 1835), pp. 77-133.

Touchard, Jean, Allegresse chrestienne de l'heureux succès des guerres de ce Royaulme et de la justice de Dieu contre les rebelles au Roy, Paris, 1572.

Turenne, Henri de La Tour d'Auvergne, vicomte, later duc de Bouillon, Mémoires, ed. Gustave Baguenault de Puchesse, Paris, 1901.

Verstegan, Richard, Théâtre des cruautés des hérétiques de notre temps [1587], ed. Frank Lestringant, Paris, 1995.

Vigor, Simon, Sermons catholiques sur les Dimenches et festes depuis l'octave de Pasques jusques à l'Advent, 2 vols, Paris, 1587.

Vindiciae contra tyrannos: sive, de Principis in Populum, Populique in Principem, legitima potestate, Stephano Junio Bruto Celta auctore, Edinburgh, 1579. Anonymous French trans., De la puissance legitime du Prince sur le peuple et $d u$ peuple sur le Prince, n.p. There is a critical ed. by Arlette Jouanna, Jean Perrin, Marguerite Soulié, André Tournon and Henri Weber, Geneva, 1979.

Viret, Pierre Viret, Le Monde à l'empire et le monde démoniacle, Geneva, 1550; 1561 edition also Geneva.

Voisin de la Popelinière, Henri Lancelot, L'Histoire de France, enrichie des plus notables occurrances survenues ez provinces de l'Europe et pays voisins, depuis l'an 1550 jusques à ces temps, 2 vols, n.p., 1581.

Weiss, Nathanël, 'L’Amiral et la Saint-Barthélemy, lettres et pièces inédites (1572)', BSHPF, 36 (1887), pp. 412-418.

-, 'La Saint-Barthélemy. Nouveaux textes et notes bibliographiques', BSHPF, 43, (1894), pp. 426-444.

\section{Secondary works}

Anon., 'Les victimes de la Saint-Barthélemy à Paris. Essai d'une topographie et d'une nomenclature des massacres d'après les documents contemporains', BSHPF, 10 (1860), pp. 34-44.

Acerra, Martine and Martinière, Guy, eds, Coligny, les protestants et la mer, Paris, 1997.

L'Amiral de Coligny et son temps, Paris, 1974.

Angelo, Vladimir, Les Curés de Paris au xvie siècle, Paris, 2005.

Babelon, Jean-Pierre, Henri IV, Paris, 1982.

Baguenault de la Puchesse, Gustave, Jean de Morvillier, évêque d'Orléans, garde des sceaux de France, Paris, 1870. 
Balsamo, Jean, Les Rencontres des Muses: italianisme et anti-italianisme dans les lettres françaises de la fin du xvi siècle, Geneva, 1992.

Baulant, Michèle and Meuvret, Jean, Prix des céréales extraits de la mercuriale de Paris (1520-1698), Paris, 1960, vol. 1.

Baury, Roger, 'Célébration de la paix de Vervins et propagande royale', in JeanFrançois Labourdette, Jean-Pierre Poussou and Marie-Catherine Vignal, eds, Le Traité de Vervins (Paris, 200o), pp. 347-372.

Benedict, Philip, 'The Saint Bartholomew's massacres in the provinces', The Historical Journal, 21 (1978), pp. 205-225.

-, Rouen during the wars of religion, Cambridge, 1981.

-, The Huguenot population of France, 1600-1685. The demographic fate and customs of a religious minority, Philadelphia, Transactions of the American Philosophical Society, 1991.

Boltanski, Ariane, Les Ducs de Nevers et létat royal. Genèse d'un compromis (vers 1550-vers 1620), Geneva, 2006.

Bordier, Henri-Léonard, La Saint-Barthélemy et la critique moderne, Geneva, 1879.

Boucher, Jacqueline, La Cour de Henri III, Rennes, 1986.

-, Société et mentalités autour de Henri III, Paris, 2007.

Bourgeon, Jean-Louis, 'Les légendes ont la vie dure: à propos de la Saint-Barthélemy et de quelques livres récents', RHMC, 34 (1987), pp. 102-116.

-, 'Une source sur la Saint-Barthélemy: l'Histoire de Monsieur de Thou relue et décryptée', BSHPF, 124 (1988), pp. 499-537.

—, 'Pour une histoire, enfin, de la Saint-Barthélemy', $R H, 282$ (1989), pp. 83-142

—, 'La Fronde parlementaire à la veille de la Saint-Barthélemy', Bibliothèque de l'Ecole des chartes, 148 (1990), pp. 17-89.

-, L'Assassinat de Coligny, Geneva, 1992.

-, 'Quand la foi était révolutionnaire: les sermons d'un curé parisien, Simon Vigor, en 1570-1572', in Mélanges offerts à Pierre Chaunu. La vie, la mort, la foi, le temps (Paris, 1993), pp. 471-484.

-, Charles IX devant la Saint-Barthélemy, Geneva, 1995.

Bourquin, Laurent, Noblesse seconde et pouvoir en Champagne aux xvi et xvii siècles, Paris, 1994.

Braudel, Fernand, La Mediterranée et le monde mediterranéen à l'époque de Philippe II, 2nd ed., 2 vols, Paris 1966. English trans. The Mediterranean and the mediterranean world in the age of Philip II, 2 vols, London, 1973.

Brunet, Serge, 'Anatomie des réseaux ligueurs dans le Sud-Ouest de la France (vers 1562-vers 1610)', in Nicole Lemaître, ed., Religion et politique dans les sociétés du Midi (Paris, 2002), pp. 153-191.

-, 'De l'Espagnol dedans le ventre!' Les catholiques du Sud-Ouest de la France face à la Réforme (vers 1540-1589), Paris, 2007.

Carroll, Stuart, Noble power during the wars of religion. The Guise affinity and the Catholic cause in Normandy, Cambridge, 1998.

Cassan, Michel, Le Temps des guerres de religion. Le cas du Limousin (vers 1530-vers 1630), Paris, 1996.

Cazauran, Nicole, 'Échos d'un massacre', in Marguerite Soulié and Robert Aulotte, eds, La Littérature de la Renaissance. Mélanges d'histoire et de critique littéraire 
offerts à Henri Weber (Geneva, 1984), pp. 239-261.

Céard, Jean, 'Les visages de la royauté en France', in Emmanuel Le Roy Ladurie, ed., Les Monarchies (Paris, 1986), pp. 73-89.

Champion, Pierre, Charles IX. La France et le contrôle de l'Espagne, 2 vols, Paris, 1939.

Châtellier, Louis, Le Catholicisme en France, 1500-1650, vol. 1: le xvi siècle, Paris, 1995.

Chaunu, Pierre, 'L'État', in Fernand Braudel and Ernest Labrousse, eds, Histoire économique et sociale de la France, vol. 1 (Paris, 1977), tome I, pp. 9-228.

Chevallier, Pierre, Henri III, Paris, 1985.

Christin, Olivier, Une révolution symbolique. L'iconoclasme huguenot et la reconstruction catholique, Paris, 1991.

—, La Paix de religion. L'autonomisation de la raison politique au xvie siècle, Paris, 1997.

-, 'Amis, frères et concitoyens. Ceux qui refusèrent la Saint-Barthélemy (1572)', Cahiers de la Villa Gillet, 11 (Sept., 200o), pp. 71-94.

Christol, Marguerite, 'La dépouille de Gaspard de Coligny', BSHPF, 111 (1965), pp. 136-140.

Church, William Farr, Constitutional thought in sixteenth-century France, Cambridge, Mass., 1941.

Cloulas, Ivan, Henri II, Paris, 1985.

-, Catherine de Medicis, Paris, 1979.

Cocula, Anne-Marie, Brantôme. Amour et gloire au temps des Valois, Paris, 1986.

-, 'Regard sur les événements nocturnes des guerres de religion', in Dominique Bertrand, ed., Penser la nuit, $x v^{e}-x v i i^{e}$ siècles (Paris, 2003), pp. 464-485.

Collins, James B., 'La guerre de la Ligue et le bien public', in Jean-François Labourdette, Jean-Pierre Poussou and Marie-Catherine Vignal, eds, Le Traité de Vervins (Paris, 2000), pp. 81-95.

Constant, Jean-Marie, Les Guise, Paris, 1984.

-, Les Français pendant les guerres de religion, Paris, 2002.

Cornette, Joël, Le Roi de guerre. Essai sur la souveraineté dans la France du grand siècle, Paris, 1993.

Cosandey, Fanny and Descimon, Robert, L'Absolutisme en France. Histoire et historiographie, Paris, 2002.

Crété, Liliane, Coligny, Paris, 1985.

Crouzet, Denis, Les Guerriers de Dieu. La violence au temps des troubles de religion, vers 1525-vers 1610, 2 vols, Seyssel, 1990.

-, 'Désir de mort et puissance absolue de Charles VIII à Henri IV', Revue de Synthèse, (1991), pp. 423-441.

—, La Nuit de la Saint-Barthélemy. Un rêve perdu de la Renaissance, Paris, 1994.

—, 'Charles IX, ou le roi sanglant malgré lui?', BSHPF (1995), pp. 323-339.

—, 'Capital identitaire et engagement religieux: aux origines de l'engagement militant de la maison de Guise, ou le tournant des années 1524-1525', in Joël Fouilleron, Guy Le Thiec and Henri Michel, eds, Sociétés et idéologies des Temps modernes. Hommage à Arlette Jouanna, 2 vols (Montpellier, 1996), vol. 2, pp. 573-589. 
—, 'La nuit de la Saint-Barthélemy: confirmations et compléments', in Chantal Grell and Arnaud Ramière de Fortanier, eds, Le Second ordre: l'idéal nobiliaire. Hommage à Ellery Schalk (Paris, 1999), pp. 55-81.

-, La Sagesse et le malheur. Michel de L'Hospital, chancelier de France, Seyssel, 2002.

—, 'Le devoir d'obéissance à Dieu: imaginaire du pouvoir royal', Nouvelle Revue $d u$ XVI Siècle, 21 (2004), pp. 19-47 (special thematic issue: Métaphysique et politique de l'obéissance dans la France du xvi siècle), pp. 19-47.

-, Le Haut Cour de Catherine de Medicis. Une raison politique aux temps de la Saint-Barthélemy, Paris, 2005.

Dareste, Rodolphe, 'François Hotman. Sa vie et sa correspondance', RH, 2 (1876), pp. $1-59$ and $367-435$.

Daubresse, Sylvie, Le Parlement de Paris ou la Voix de la raison (1559-1589), Geneva, 2005.

Daussy, Hugues, Les Huguenots et le roi. Le combat politique de Philippe Duplessis-Mornay, Geneva, 2002.

Davies, Joan Margaret, 'Languedoc and its governeur. Henri de Montmorency-Damville, 1563-1589', unpublished Ph.D dissertation, University of London, 1974.

Davis, Natalie Zemon, Society and culture in early modern France, Stanford, Calif., 1975.

Decrue, Francis, Le Parti des politiques au lendemain de la Saint-Barthélemy, Paris, 1892.

Delaborde, Jules, Gaspard de Coligny, amiral de France, 3 vols, Paris, 1879-82.

Delumeau, Jean, Le Péché et la peur, Paris, 1983.

-, Rassurer et protéger, Paris, 1989.

Delumeau, Jean, and Wanegffelen, Thierry, Naissance et affirmation de la Réforme, $8^{\mathrm{e}}$ ed., Paris, 1997.

Denis, Anne, 'La Saint-Barthélemy vue et jugée par les Italiens', in Danielle Boillet and Corinne Lucas-Fiorato, eds, L'Actualité et sa mise en écriture dans l'Italie de la Renaissance (Paris, Centre interuniversitaire de recherches sur la Renaissance italienne, vol. 26, 2005), pp. 202-225.

Descimon, Robert, 'Solidarité communautaire et sociabilité armée: les compagnies de la milice bourgeoise à Paris (xvi ${ }^{\mathrm{e}}-\mathrm{xvii}^{\mathrm{e}}$ siècles)', in Françoise Thélamon, ed., Sociabilité, pouvoirs et société (Rouen, 1987), pp. 599-610.

-, 'Paris on the eve of Saint Bartholomew: Taxation, privilege and social geography', in Philip Benedict, ed., Cities and social change in early modern France (London, 1989), pp. 69-104.

Diefendorf, Barbara, Paris city councillors in the sixteenth century: the politics of patrimony, Princeton, 1983.

—, 'Prologue to a massacre: popular unrest in Paris, 1557-1572', American Historical Review, 90 (1985), pp. 1067-91.

—, 'Les divisions religieuses dans les familles parisiennes avant la Saint-Barthélemy', HES, 7 (1988), pp. 55-77.

-, Beneath the cross. Catholics and Huguenots in sixteenth-century Paris, New York and Oxford, 1991. 
—, 'La Saint-Barthélemy et la bourgeoisie parisienne', HES, 17 (1998), pp. 341-352.

-, The Saint Barthlomew's Day massacre. A brief history with documents, Boston and New York, 2009.

Dubost, Jean-François, La France italienne, $x v i^{e}-x v i i^{e}$ siècle, Paris, 1997.

Dufour, Alain, 'Le colloque de Poissy', in Mélanges d'histoire du xvi siècle offerts à Henri Meylan (Geneva, 1970), pp. 127-137.

-, Théodore de Bèze, poète et théologien, Geneva, 2006.

El Kenz, David, Les Bûchers du roi. La culture protestante des martyrs (1523-1572), Seyssel, 1997.

-, 'La victime catholique au temps des guerres de religion. La sacralisation du prêtre', in Benoît Garnot, ed., Les Victimes, des oubliées de l'Histoire? (Rennes, 2000), pp. 192-199.

—, 'La Saint-Barthélemy à Dijon: un non-événement?', Annales de Bourgogne, 74 (2002), pp. 139-157.

- , 'Les usages subversifs du martyre dans la France des troubles de religion: de la parole au geste', Revue des sciences humaines, 269 (2003) [Martyrs et martyrologes, eds Frank Lestringant and Pierre-François Moreau], pp. 33-51.

El Kenz, David, ed., Le Massacre, objet d'histoire, Paris, 2005.

Engammare, Max, L'Ordre du temps. L'invention de la ponctualité au xvi siècle, Geneva, 2004.

Erlanger, Philippe, Le Massacre de la Saint-Barthélemy, Paris, 1960. English trans. The Saint Bartholomew's night, London 1962.

Fazy, Henri, La Saint-Barthélemy et Genève, étude historique (Mémoires de l'Institut national genevois, vol. 14). Geneva, 1879.

Foa, Jérémie, 'Making peace: the commissions for enforcing the pacification edicts in the reign of Charles IX (156o-1574)', French History, 18 (2004), pp. 256-274.

Forestié, Edouard, Un Capitaine gascon du xvie siècle: Corbeyran de Cardaillac-Sarlabous, Paris, 1897.

Forsyth Elliott, Christopher, La Justice de Dieu. Les Tragiques d'Agrippa d'Aubigné et la Réforme protestante en France au xvie siècle, Paris, 2005.

Fragonard, Marie-Madeleine, 'Létablissement de la raison d'état et la SaintBarthélemy', in Miroirs de la Raison d'état (Cahiers de du Centre de recherches historiques, no 20 1998), pp. 49-65.

—, La Pensée religieuse d'Agrippa d'Aubigné et son expression, 1981, rev. ed., Paris, 2004.

Froeschlé-Chopard, Marie-Hélène, Dieu pour tous et Dieu pour soi. Histoire des confréries et de leurs images à l'époque moderne, Paris, 2007.

Garrisson, Janine, Tocsin pour un massacre, ou la saison des Saint-Barthélemy, Paris, 1968.

—, La Saint-Barthélemy, Paris, 1987.

—, Protestants du Midi, 1559-1598, Toulouse, 1980, reprint. 1991.

—, Les Derniers Valois, Paris, 2001.

Gauchet, Marcel, Le Désenchantement du monde: une histoire politique de la religion, Paris, 1985. English trans., The Disenchantment of the world, Princeton, 1997.

Gelderen, Martin van, The Political thought of the Dutch revolt, 1555-1590, Cambridge, 1992. 
Germa-Romann, Hélène, Du 'bel mourir' au 'bien mourir'. Le sentiment de la mort chez les gentilshommes français (1515-1643), Geneva, 2001.

Gigon, Stéphane-Claude, La Troisième guerre de religion. Jarnac-Moncontour (1568-1569), Paris, 1911.

Girard, René, La Violence et le Sacré, Paris, 1972. English trans., Violence and the sacred, Baltimore, 1977.

Greengrass, Mark, 'War, politics and religion in Languedoc during the government of Henri de Montmorency-Damville (1574-1610), unpublished D.Phil dissertation, University of Oxford, 1979.

- , 'Functions and limits of political clientelism in France before Cardinal Richelieu', in Neithard Bulst, Robert Descimon and Alain Guerreau, eds, L'État ou le roi. Les fondations de la modernité monarchique en France (xiv ${ }^{e}-x v i i^{e}$ siècles) (Paris, 1996), pp. 69-82.

-, 'Amnistie et oubliance: un discours politique autour des édits de pacification pendant les guerres de religion', in Paul Mironneau and Isabelle Péray-Clottes, eds, Paix des armes, paix des âmes (Paris, 200o), pp. 113-123.

-, 'Pluralism and equality: the peace of Monsieur, May 1576', in Keith Cameron, Mark Greengrass and Penny Roberts, eds, The Adventure of religious pluralism in early modern France (Bern, 2000), pp. 45-63.

Greffe, Florence and Lothe, José, La vie, les livres et les lectures de Pierre de L'Estoile, Paris, 2004.

Halévi, Ran, 'Le testament de la royauté. L'éducation politique de Louis XVI', in Ran Halévi, ed., Le Savoir du Prince (Paris, 2002), pp. 311-361.

Haran, Alexandre Y., Le Lys et le globe: messianisme dynastique et rêve impérial en France aux $x v i^{e}$ et $x v i i^{e}$ siècles, Seyssel, 2000.

Hauser, Henri, François de La Noue, Paris, 1892.

—, 'Le père Edmond Auger et le massacre de Bordeaux, 1572', BSHPF, 6o (1911), pp. 289-306.

-, Les Sources de l'histoire de France, xvie siècle, 4 vols (Paris, 1912), vol. 3, Les guerres de Religion (1559-1589).

Heller, Henry, Anti-Italianism in sixteenth-century France, Toronto, 2002.

Higman, Francis, La Diffusion de la Réforme en France, 1520-1565, Geneva, 1992.

Hillairet, Jacques, Dictionnaire historique des rues de Paris, 2 vols, 1oth ed., Paris, 1997.

Holt, Mack P., The Duke of Anjou and the politique struggle during the wars of religion, Cambridge, 1986.

-, 'Attitudes of the French nobility at the Estates-General of 1576', SCJ, 18 (1987), pp. 489-504.

-, The French wars of religion, 1562-1629, Cambridge, 1995.

Huchard, Cécile, D'Encre et de sang. Simon Goulart et la Saint-Barthélemy, Paris, 2007.

Jacquiot, Josèphe, 'Medailles et jetons commémorant la Saint-Barthélemy', Revue d'histoire littéraire de la France (1973), pp. 784-793.

Jouanna, Arlette, Le Devoir de révolte. La noblesse française et la gestation de l'État moderne, 1559-1661, Paris, 1989.

—, 'Être "bon Français" au temps des guerres de religion: du citoyen au sujet', in 
Ouzi Élyada and Jacques Le Brun, eds, Conflits politiques, controverses religieuses. Essais d'histoire européennes aux $16^{e}-18^{e}$ siècles (Paris, 2002), pp. 19-32.

-, "Capituler avec son prince": la question de la contractualisation de la loi au xvi ${ }^{\mathrm{e}}$ siècle', in Paul-Alexis Mellet, ed., 'Et de sa bouche sortait un glaive.' Les monarchomaques au xvi siècle (Geneva, 2006), pp. 131-143.

Jouanna, Arlette, Boucher, Jacqueline, Biloghi, Dominique and Le Thiec, Guy, Histoire et dictionnaire des guerres de religion, Paris, 1998.

Joutard, Philippe, Estèbe [Garrison], Janine, Labrousse, Élisabeth and Lecuir, Jean, La Saint-Barthélemy ou les résonances d'un massacre, Neuchâtel, 1976.

Kantorowicz, Ernst, The King's two bodies. A study in medieval political theology, Princeton, 1957. French trans., Les Deux corps du roi. Essai sur la théologie politique au Moyen Âge, Paris, 1989.

Kelley, Donald R., The Beginning of ideology: Consciousness and society in the French reformation, Cambridge, 1981.

Kervyn de Lettenhove, Joseph, Les Huguenots et les gueux, 6 vols, Bruges, 1883-85.

Kingdon, Robert M., Myths about the St. Bartholomew's day massacres, 1572-1576, Cambridge, Mass., 1988.

-, Church and society in Reformation Europe, London, 1985.

Knecht, Robert Jean, Catherine de' Medici, London, 1998. French trans., Catherine de Medicis (1519-1589), Brussels, 2003.

-, The French civil wars, 1562-1598, Harlow, 2000.

Konnert, Mark, Civic agendas as religious passion: Châlons-sur-Marne during the French wars of religion, Kirksville, Miss., 1997.

—, 'La tolérance religieuse en Europe aux $\mathrm{xvi}^{\mathrm{e}}$ et xvii $\mathrm{e}^{\mathrm{e}}$ siècles. Une approche issue de la psychologie sociale et de la sociologie', in Thierry Wanegffelen, ed., De Michel de L'Hospital à l'edit de Nantes. Politique et religion face aux églises (Clermont-Ferrand, 2002), pp. 97-113.

Krynen, Jacques, L'Empire du roi. Idées et croyances politiques en France, $x i i i^{e}-x v^{e}$ siècle, Paris, 1993.

La Ferrière, Hector de, 'Les dernières conspirations du règne de Charles IX', Revue des questions historiques, 48 (1890), pp. 421-470.

-, La Saint-Barthélemy, la veille, le jour, le lendemain, Paris, 1892.

Lazard, Madeleine, Pierre de Bourdeille, seigneur de Brantôme, Paris, 1995.

Le Gall, Jean-Marie, Le Mythe de Saint Denis entre Renaissance et Révolution, Seyssel, 2007.

Le Roux, Nicolas, La Faveur du roi. Mignons et courtisans au temps des derniers Valois (vers 1547-vers 1589), Seyssel, 2000.

—, 'La Saint-Barthélemy des Italiens n'aura pas lieu: un discours envoyé à Catherine de Medicis en 1573', in Bernard Barbiche, Jean-Pierre Poussou and Alain Tallon, eds, Pouvoirs, contestations et comportements dans l'Europe moderne. Mélanges en l'honneur du professeur Yves-Marie Bercé (Paris, 2005), pp. 165-183.

—, Un Régicide au nom de Dieu. L'Assassinat d'Henri III, Paris, 2006.

-, Les Guerres de religion, 1559-1629, Paris, 2009.

Le Roy Ladurie, Emmanuel, L'État royal de Louis XII à Henri IV, 1460-1610, Paris, 1987. English trans., The Royal French state 1460-1610, Oxford, 1994.

Lecoq, Anne-Marie, François $1^{\text {er }}$ imaginaire, Paris, 1987. 
Lemaitre, Nicole, Saint Pie V, Paris, 1994.

Lestringant, Frank, Agrippa d'Aubigné. Les Tragiques, Paris, 1986.

—, La Cause des martyrs dans les Tragiques d'Agrippa d'Aubigné, Mont-de-Marsan, 1991.

—, Lumière des martyrs. Essai sur le martyre au siècle des réformes, Paris, 2004.

Levene, Mark and Roberts, Penny, eds, The massacre in history, New York, 1999.

Manesch, Scott M., Theodore Beza and the quest for peace in France, 1572-1598, Leiden, 2000.

Mariéjol, Jean-H., La Réforme et la Ligue. L'edit de Nantes (1559-1598) (Histoire de France des origines à la Révolution, ed. Ernest Lavisse, vol. 6), Paris, 1904, reprint Paris, 1983.

Mastellone, Salvo, Venalità e machiavellismo in Francia, 1572-1610: all'origine della mentalità politica borghese, Florence, 1972.

Mellet, Paul-Alexis, ed., 'Et de sa bouche sortait un glaive.' Les monarchomaques au $x v i^{e}$ siècle, Geneva, 2006.

-, Les Traités monarchomaques. Confusion des temps, résistance armée et monarchie parfaite (1560-1600), Geneva, 2007.

Mieck, Ilja, 'Die Bartholomäusnacht als Forschungsproblem: Kritische Bestandsaufnahme und neue Aspekte', Historische Zeitschrift, 216 (1973), pp. 73-110.

Monod, Henri, 'Un document sur la Saint-Barthélemy', Revue de Paris, 4 (1908), pp. 770-794.

—, 'La version du duc d'Anjou sur la Saint-Barthélemy', RH, 101 (1909), pp. 316-325.

Mousnier, Roland, L'Assassinat d'Henri IV. Le problème du tyrannicide et l'affermissement de la monarchie absolue, Paris, 1964.

Muchembled, Robert, Passions de femmes au temps de la reine Margot, 1553-1615, Paris, 2003.

Nakam, Géralde, Au lendemain de la Saint-Barthélemy. Jean de Léry, Histoire memorable du siège de Sancerre, Paris, 1975.

—, Les Essais de Montaigne, miroir et procès de leur temps. Témoignage historique et création littéraire, revised ed., Paris, 2001.

—, 'Le rire de Coligny', in Géralde Nakam, Chemins de la Renaissance (Paris, 2005), pp. 193-212.

Nicollier, Béatrice, Hubert Languet, 1518-1581. Un réseau politique international, de Melanchthon à Guillaume d'Orange, Geneva, 1995.

Noailles, Emmanuel de, Henri de Valois et la Pologne en 1572, 2 vols, Paris, 1867.

Pablo, Jean de, 'Contribution à l'étude de l'histoire des institutions militaires huguenots. II. L'armée huguenote entre 1562 et 1573', Archiv für Reformationgeschichte, 48 (1957), pp. 192-216.

Paz, Julián, Archivo general de Simancas. Catálogo IV. Secretaría de Estado (Capitulaciones con Francia y negociaciones diplomáticas de los embajadores de España en aquella corte, seguido de un serie cronologica de éstos), vol. 1 (12251714), Madrid, 1814.

Peschot, Bernard, 'Alexandre de Bessot de Lamothe (1823-1897) et les protestants du Midi', in Joël Fouilleron and Henri Michel, eds, Mélanges Michel Péronnet, vol. 2, La Réforme, (Montpellier, 2003), pp. 313-332.

Petris, Loris, La Plume et la Tribune. Michel de L'Hospital et ses discours (1559- 
1562), Geneva, 2002.

Picot, Georges, Histoire des États généraux, $2^{\mathrm{e}}$ ed., 3 vols, Paris, 1888.

Pineaux, Jacques, La Poésie des protestants de langue française (1559-1598), Paris, 1971.

Poncet, Olivier, Pomponne de Bellièvre (1529-1607). Un homme d'état au temps des guerres de religion, Paris, 1998.

Poujol, Jacques, 'Étymologies légendaires des mots France et Gaule pendant la Renaissance', Publications of the Modern Language Association of America, 72 (1957), pp. 900-914.

Pradel, Charles, 'Un marchand de Paris au xvi ${ }^{\mathrm{e}}$ siècle (1560-1588)', Mémoires de l'Académie des sciences, inscriptions et belles-lettres de Toulouse, 9th series, vol. 1 (Toulouse, 1889), pp. 327-351; vol. 2 (1890), pp. 39o-427.

Renoux-Zagamé, Marie-France, 'Du juge-prêtre au roi-idole. Droit divin et constitution de l'état dans la pensée juridique française à l'aube des Temps modernes', in Jean-Louis Thireau, ed., Le Droit entre laïcisation et néo-sacralisation (Paris, 1997), pp. 143-186.

Richet, Denis, 'Aspects socioculturels des conflits religieux à Paris dans la seconde moitié du $\mathrm{xvi}^{\mathrm{e}}$ siècle', De la Réforme à la Révolution. Études sur la France moderne (Paris, 1991), pp. 15-51.

Roberts, Penny, A City in conflict. Troyes during the French wars of religion, Manchester, 1996.

Roelker, Nancy Lyman, One king, one faith. The Parlement of Paris and the religious reformations of the sixteenth century, Berkeley, 1996.

-, Queen of Navarre. Jeanne d'Albret 1528-1572, Cambridge, Mass., 1968. French trans. Jeanne d'Albret, reine de Navarre, 1528-1572, Paris, 1979.

Romier, Lucien, 'La Saint-Barthélemy. Les événements de Rome et la prémeditation du massacre', Revue du seizième siècle, 1 (1913), pp. 529-560.

Root, Hilton, La construction de l'État moderne en Europe. La France et l'Angleterre, Paris, 1994.

Samaran, Charles, 'Un humaniste italien, Guido Lolgi, témoin de la SaintBarthélemy', Studi in onore di Ricardo Filangeri (Naples, 1959), vol. 2, pp. 397-404.

Sémelin, Jacques, 'Analyser le massacre. Réflexions comparatives', Questions de recherches/Research in question, 7 (Sept. 2002), pp. 1-42. Also available in electronic form at http://www.ceri-sciences-po.org.

-, Purifier et détruire. Usages politiques des massacres et génocides, Paris, 2005.

Senellart, Michel, Machiavélisme et raison d'Etat, Paris, 1989.

Simonin, Michel, Pierre de Ronsard, Paris, 1990.

-, Vivre de sa plume au xvie siècle, ou la carrière de François de Belleforest, Geneva, 1992.

-, Charles IX, Paris, 1995.

Smith, Marc, 'Familiarité française et politesse italienne au $\mathrm{xvi}^{\mathrm{e}}$ siècle. Les diplomates italiens juges des manières de la cour des Valois', RHD 102 (1988), pp. 193-232.

Solnon, Jean-François, Catherine de Médicis, Paris, 2003.

Soman, Alfred, ed., The Massacre of St. Bartholomew. Reappraisals and documents, 
The Hague, 1974.

Soulié, Marguerite, 'La Saint-Barthélemy et la réflexion sur le pouvoir', in Franco Simone, ed., Culture et politique en France à l'époque de l'humanisme et de la Renaissance (Turin, 1974), pp. 413-425.

—, L'Inspiration biblique dans la poésie religieuse d'Agrippa d'Aubigné, Paris, 1977. Souriac, Pierre-Jean, Une guerre civile. Affrontements religieux et militaires dans le Midi toulousain, 1562-1596, Seyssel, 2008.

Sutherland, N. M., The Massacre of St Bartholomew and the European conflict 1559-1572, London, 1973.

-, The Huguenot struggle for recognition, New Haven and London, 1980.

-, 'Le massacre de la Saint-Barthélemy: la valeur des témoignages et leur interprétation', RHMC, 38 (1991), pp. 529-554.

Sypher, Wylie, “Faisant ce qu'il leur vient à plaisir": The image of protestantism in French Catholic polemic on the eve of the religious wars', SCJ, 11 (1980), pp. 59-84.

Tallon, Alain, La France et le concile de Trente, Rome, 1997.

-, Conscience nationale et sentiment religieux en France au xvi siècle, Paris, 2002.

Taylor, Larissa Juliet, Heresy and orthodoxy in sixteenth-century Paris. François Le Picart and the beginnings of the Catholic Reformation, Leiden, 1999.

Thuau, Étienne, Raison d'état et pensée politique à l'époque de Richelieu, Athens, 1966, reprint Paris, 2000.

Tournon, André, 'La poétique du témoignage dans Les Tragiques d'Agrippa d'Aubigné', in Olivier Pot, ed., Poétiques d’Aubigné (Geneva, 1999), pp. 135-146.

Turchetti, Mario, Concordia o tolleranza? François Bauduin (1520-1573) e $i$ 'moyenneurs', Geneva, 1984.

-, Tyrannie et tyrannicide de l'Antiquité à nos jours, Paris, 2001.

-, 'L'arrière-plan politique de l'edit de Nantes, avec un aperçu de l'anonyme De la concorde de l'Estat par l'observation des Edicts de pacification', in Michel Grandjean and Bernard Roussel, eds, Coexister dans l'intolérance. L'edit de Nantes (1598), (Geneva, 1998), pp. 93-114.

Vasquez de Prada, Valentin, Felipe II y Francia. Política, religión y razón de Estado, Pamplona, 2004.

Venard, Marc, 'La présentation de la Saint-Barthélemy aux Polonais en vue de l'élection d'Henri de Valois', in Les Contacts religieux franco-polonais du Moyen-Âge à nos jours (Paris, 1985), pp. 116-127.

—, 'Arrêtez le massacre!', RHMC, 39 (1992), pp. 645-661.

-, Réforme protestante, Réforme catholique dans la province d'Avignon, xvi siècle, Paris, 1993.

Viennot, Éliane, Marguerite de Valois. Histoire d'une femme, histoire d'un mythe, Paris, 1993.

—, 'À propos de la Saint-Barthélemy et des Mémoires de Marguerite de Valois', Revue d'Histoire Littéraire de la France (Sept.-Oct. 1996), pp. 894-917.

Vivanti, Corrado, Lotta politica e pace religiosa in Francia tra Cinque e Seicento, Turin 1963. French trans., Guerre civile et paix religieuse dans la France d'Henri $I V$, Paris, 2006.

Wanegffelen, Thierry, Ni Rome ni Genève. Des fidèles entre deux chaires en France 
au $x v i^{e}$ siècle, Paris, 1997.

-, Catherine de Medicis. Le pouvoir au féminin, Paris, 2005.

Weiss, Nathanaël, 'La Seine et le nombre des victimes parisiennes de la SaintBarthélemy', BSHPF, 46 (1897), pp. 474-481.

Wood, James B., 'The Royal army during the wars of religion, 1559-1576', in Mack P. Holt, ed., Society and institutions in early modern France (Athens, Georgia, 1991), pp. 1-35.

-, The King's army. Warfare, soldiers and society during the wars of religion in France, 1562-1576, Cambridge, 1996.

Yardeni, Myriam, La Conscience nationale en France pendant les guerres de religion (1559-1598), Paris-Brussels, 1971.

Yates, Frances A., Astraea. The imperial theme in the sixteenth century. London 1975. French trans., Astrée. Le symbolisme impérial au xvie siècle, Paris, 1989.

-, The French academies of the sixteenth century, London, 1947. French trans., Les Académies en France au xvie siècle, Paris, 1996. 
Arlette Jouanna - 9781526112194

Downloaded from manchesterhive.com at $04 / 26 / 2023$ 09:14:23AM 